\title{
Bayesian Inference Supports the Use of Bypass Surgery Over Percutaneous Coronary Intervention To Reduce Mortality in Diabetic Patients with Multivessel Coronary Disease
}

\author{
Christopher D. Lang ${ }^{1}$, Yulei He $\mathrm{H}^{2, \#}$ and John A. Bittl ${ }^{3, *}$ \\ ${ }^{1}$ Alpert School of Medicine, Brown University, Providence, RI, USA \\ ${ }^{2}$ Office of Research and Methodology, National Center for Health Statistics, Centers for Disease Control and \\ Prevention, Hyattsville, MD, USA
}

${ }^{3}$ Munroe Regional Medical Center, Ocala, FL, USA

\begin{abstract}
Background: Coronary artery bypass graft (CABG) surgery may confer a survival advantage over percutaneous coronary intervention (PCI) in diabetic patients with multivessel coronary artery disease (CAD), but results of individual studies have been mixed. The primary aim of the current study was to compare mortality rates in diabetic patients with multivessel CAD randomized to either or $C A B G$ or $\mathrm{PCl}$ at 5 years or longest follow-up.
\end{abstract}

Methods: Using a Bayesian approach, we updated a prior probability distribution elicited from 8 clinical trials $(N=2024)$ with the likelihood obtained from the Future Revascularization Evaluation in Patients with Diabetes Mellitus: Optimal Management of Multivessel Disease (FREEDOM) $(N=1460)$ to determine whether clinical trial evidence supports the underlying hypothesis that $\mathrm{CABG}$ is superior to $\mathrm{PCI}$ for diabetics with multivessel CAD.

Results: A conjugate normal model comparing mortality rates favored the use of CABG (posterior mean odds ratio [OR] $=0.58,95 \%$ Bayesian credible interval $[\mathrm{BCl}]=0.48-0.71)$. Models weighted by the use of drug-eluting stents also favored the use of CABG over $\mathrm{PCl}(O R=0.61,95 \% \mathrm{BCl} 0.48-0.78)$, as did models weighted by study age $(O R=0.64$, $95 \% \mathrm{BCl} 0.52-0.80)$ or use of arterial conduits $(O R=0.64,95 \% \mathrm{BCl} 0.51-0.81)$. The results were supported by a Bayesian hierarchical meta-analysis using a non-informative prior distribution $(O R=0.55,95 \% \mathrm{BCl} 0.37-0.76)$.

Conclusions: By integrating evidence from various studies, Bayesian methods directly support the underlying hypothesis that revascularization with $C A B G$ improves survival compared with $\mathrm{PCl}$ in diabetic patients with multivessel $\mathrm{CAD}$.

Keywords: Health policy and outcome research, catheter-based coronary interventions, stents, CV surgery, coronary artery disease, diabetes mellitus.

Patients with diabetes mellitus (DM) have more diffuse coronary artery disease (CAD) than nondiabetics and stronger evidence of a prothrombotic state and vascular inflammation. These factors may impair the response to percutaneous coronary intervention (PCl) [1], as compared with surgical revascularization. A subgroup analysis from the Bypass Angioplasty Revascularization Investigation (BARI) [2] reported that diabetic patients treated with coronary artery bypass graft (CABG) surgery had lower mortality rates at 5 years than those treated with PTCA $(5.8 \%$ versus $20.6 \%, P=0.0003$ ).

Randomized controlled trials (RCTs) comparing CABG with $\mathrm{PCl}$ for diabetic patients with multivessel $C A D$ have produced mixed findings, though none have suggested a survival advantage of $\mathrm{PCl}$. The Future Revascularization Evaluation in Patients with Diabetes

\footnotetext{
*Address correspondence to this author at the Munroe Regional Medical Center, 1221 SE $5^{\text {th }}$ Street, Ocala, FL, USA; Tel: 352-351-7206; Fax: 352-4025399; E-mail: jabittl@mac.com

\#The project was conceived while Yulei He was at the Harvard Medical School. The findings and conclusions in this paper are those of the authors and do not necessarily represent the official position of the National Center for Health Statistics.
}

Mellitus: Optimal Management of Multivessel Disease (FREEDOM) trial randomized diabetic patients with multivessel CAD to either CABG or PCl and reported lower 5-year mortality after surgical revascularization $(11.0 \%$ vs. $16.3 \% ; \quad P=0.049)$ [3]. Although the FREEDOM trial was the first large trial in diabetic patients with multivessel CAD, it was not powered to detect a difference in all-cause mortality [3].

A meta-analysis of 8 trials [4], including FREEDOM, suggested that revascularization with $C A B G$ compared with $\mathrm{PCl}$ in diabetic patients with multivessel $\mathrm{CAD}$ decreased all-cause mortality by one third at 5 years or longest follow-up (relative risk $[R R]$ 0.67, 95\% confidence intervals [Cl] 0.52-0.86). After most of the older studies contained in the meta-analysis were reported, several advances in surgical and interventional practice have appeared. To determine whether newer stents have an advantage over older interventional approaches, Bangalore and colleagues [5] performed a network meta-analysis and reported that the use of cobalt-chromium everolimus-eluting stents may narrow the mortality gap between CABG and $\mathrm{PCl}(R R=0.90,95 \% \mathrm{Cl} 0.54-1.49)$. On the other 
hand, Hakeem and colleagues [6] performed a metaanalysis of contemporary trials and reported that CABG in diabetic patients with multivessel CAD was associated with lower mortality than the use of drugeluting stents (DES, $R R=0.66,95 \% \mathrm{Cl} 0.48-0.21$ ).

The primary aim of the current study was to compare mortality rates in diabetic patients with multivessel $C A D$ after treatment with $\mathrm{CABG}$ or $\mathrm{PCI}$. Beyond the standard techniques used for statistical analysis, the current study incorporated Bayesian methods to establish inferences based on probability functions [7]. The first step in the analysis defined a prior probability distribution elicited from 8 clinical trials [2,8-14]. The next step updated the prior with a likelihood distribution obtained from the FREEDOM trial [3] to generate the posterior distribution, which contained the parameter governing the probability that CABG is superior to $\mathrm{PCl}$ for diabetic patients with multivessel CAD. A sensitivity analysis was then developed to study the influence of stent type, study age and use of arterial conduits on the treatment effect in the posterior distribution. A final analysis used a Bayesian hierarchical approach to generate conclusions about whether CABG is superior to $\mathrm{PCI}$ in reducing mortality in diabetic patients with multivessel CAD.

\section{METHODS}

\section{Prior Distribution}

The studies for the prior distribution appeared in the data supplements accompanying the 2011 clinical guidelines for revascularization [15]. The evidence base included dedicated RCTs of diabetic patients and diabetic-subgroup analyses of RCTs.

In the current report, the comparison of CABG with $\mathrm{PCl}$ is represented by the odds ratio $(O R)$, which is the odds of death following CABG divided by the odds of death following $\mathrm{PCl}$. An $O R<1$ favors CABG.

Because 8 previous studies are available (Table 1) [2,8-14], they can be used as a basis for a prior distribution, with the understanding that the Bayesian concept of the term "prior" is not a literal chronological prerequisite [7]. In the prior studies, we have data $y_{1}, \ldots, y_{H}$, each of which is assumed to follow a normal distribution

$$
y h \sim N\left[\theta h, \sigma_{h}^{2}\right],
$$

governed by an underlying treatment parameter $\theta_{h}$ and its variance $\sigma_{h}^{2}$ for $h=1,2, \ldots, 8$ trials. If we are willing to assume that the studies are exchangeable [16], this leads to the use of a meta-analysis (Figure 1). It is common practice to make the assumption that the prior distribution $p(\theta)$ takes the form of a normal distribution [7]

$$
p(\theta)=N\left[\theta \mid \mu, \frac{\sigma^{2}}{m_{0}}\right]
$$

where $\theta$ is the unknown underlying treatment effect, $\mu$ is the prior mean, $\sigma$ is the standard deviation, and $m_{0}$ is the implicit sample size.

If $y_{1}$ observations in trial 1 have been crossclassified according to treatment received in a $2 \times 2$

\begin{tabular}{|c|c|c|c|c|c|c|c|c|c|}
\hline \multirow[b]{2}{*}{ Reference } & \multirow{2}{*}{$\begin{array}{c}\text { All } \\
\text { Patient } \\
\text { Age (yrs) }\end{array}$} & \multicolumn{2}{|c|}{ Total N } & \multicolumn{2}{|c|}{ Deaths } & \multicolumn{4}{|c|}{ Summary statistics } \\
\hline & & CABGPCI & $\mathrm{PCl}$ & $C A B G$ & $\mathrm{PCl}$ & OR & $\log _{e}(O R)$ & $V(\theta)$ & $\underset{(\sigma=2)}{m}$ \\
\hline BARI [2] & 62 & 180 & 173 & 16 & 47 & 0.267 & -1.320 & 0.093 & 43 \\
\hline ARTS [8] & 61 & 96 & 112 & 8 & 15 & 0.604 & -0.504 & 0.201 & 20 \\
\hline ERACI II [9] & 62 & 39 & 39 & 4 & 4 & 1.000 & 0.000 & 0.495 & 8 \\
\hline MASS II [10] & 60 & 59 & 56 & 9 & 9 & 0.941 & -0.061 & 0.245 & 16 \\
\hline SoS [11] & 62 & 74 & 68 & 1 & 7 & 0.167 & -1.788 & 0.828 & 5 \\
\hline CARDia [12] & 64 & 248 & 254 & 32 & 37 & 0.871 & -0.138 & 0.065 & 61 \\
\hline SYNTAX [13] & 65 & 202 & 227 & 26 & 44 & 0.616 & -0.485 & 0.070 & 58 \\
\hline VA CARDS [14] & 62 & 97 & 101 & 5 & 21 & 0.223 & -1.502 & 0.248 & 16 \\
\hline FREEDOM [3] & 63 & 761 & 699 & 83 & 114 & 0.629 & -0.463 & 0.023 & 171 \\
\hline
\end{tabular}

Table 1: Data for Pooled Bayesian Model

The $m$ 's are the "effective number of events" in a balanced trial, obtained from setting the estimated variances of the $\log _{e}(O R)$ to $\sigma^{2} / m$. The pooled results are obtained by adding the $m$ 's and weighting the $\log _{e}(O R)$ by their respective $m$ 's and labeled $n_{0}$ and used as the basis for the prior distribution for FREEDOM. 


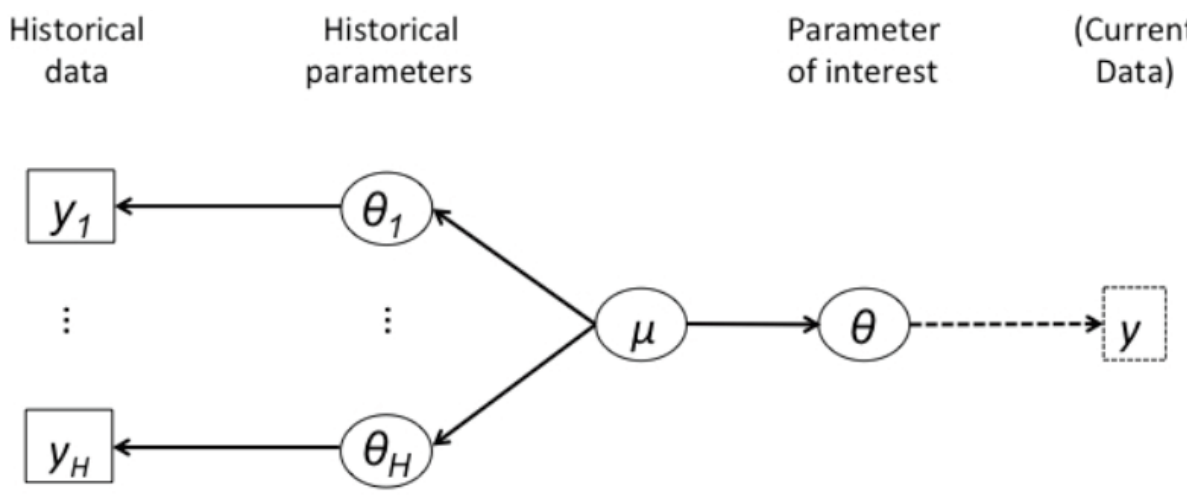

Figure 1: Exchangeable Model. The figure illustrates the assumptions relating parameters $\left(\theta_{1}, \ldots, \theta_{H}\right)$ underlying historical data $\left(y_{1}, \ldots, y_{H}\right)$ to the parameter of current interest $(\theta) . \mu$ represents the pooled log odds ratio from the historical studies, and arrows represent normal distributions.

table, and the odds of death after CABG is $a / c$ (the number of deaths divided by the number of survivors) and the odds of death after $\mathrm{PCl} b / d$, then the $O R$ describing the trial results is given by $(a / c) /(b / d)$. Because some trials have small numbers of events, we add 0.5 to both the numerator and denominators, and the trial result $\mu$ on the $\log _{\mathrm{e}}$ scale is the estimator of choice for the treatment effect [7],

$\mu_{m}=\log _{e}\left[\frac{\left(a+\frac{1}{2}\right)\left(d+\frac{1}{2}\right)}{\left(b+\frac{1}{2}\right)\left(c+\frac{1}{2}\right)}\right]$.

The estimator has an approximate variance

$V\left(\mu_{m}\right)=\frac{1}{a+\frac{1}{2}}+\frac{1}{b+\frac{1}{2}}+\frac{1}{c+\frac{1}{2}}+\frac{1}{d+\frac{1}{2}}$.

In order to put the variance into a workable form for the prior distribution, some experts recommend calculating the standard error $\sigma / \sqrt{m}$ for each study using a term $m$ to reflect the "effective number of events" in balanced trials, which is obtained from setting the variance of the $\log _{\mathrm{e}}(O R)$ to $\sigma^{2} / m$ [7]. In a $2 \times 2$ table for a balanced randomized trial, it can be assumed that the sample sizes for each treatment are approximately equal, the number of deaths $a \approx b$ are very small compared with the number of surviving patients $c \approx d$ in each treatment group, so that Eq. 4 simplifies to:

$V\left(\mu_{m}\right) \approx \frac{2}{a} \approx \frac{4}{m}$,

where $m=a+b$ is the number of events, allowing $\sigma=2$ to be an appropriate choice [7]
After calculating $m_{h}$ for each $y_{h}$, we can obtain the "pooled" results by summing the $m$ s for the $h=1,2, \ldots$, 8 trials. The pooled $m$ can be relabeled $m_{0}$ to represent the overall "effective number of events" in the prior distribution. We can use this value to calculate a pooled $\log _{\mathrm{e}}(O R)$ for the prior distribution by weighting the individual $\log _{\mathrm{e}}(O R) \mathrm{s}$ by their respective $m s$ divided by the sum $m_{0}$ [17], using the standard approach:

$\mu_{0}=\frac{\sum_{h=1}^{8} m_{h} y_{h}}{m_{0}}$

In the evaluation of evidence comprising the prior distribution, it is reasonable to down-weight early trials by adjusting the prior "number of events" from $m$ to $\alpha m$, where $\alpha$ is a normalizing factor [18]. With this approach, $\alpha$ can be set to $0,0.1,0.5$ and 1.0 , to discount earlier trials that did not use, for example, drug-eluting stents (DES) or a high proportion of left internal mammary artery (LIMA) grafts:

$$
\mu_{\text {weighted }}=\frac{\sum_{h=1}^{8} \alpha_{h} m_{h} y_{h}}{\sum_{h=1}^{8} \alpha_{h} m_{h}}
$$

\section{Likelihood}

In the context of a contemporary randomized clinical trial such as FREEDOM [3], it is reasonable to assume that the data can be summarized by a statistic, $y_{n}=\log _{e}\left(O R_{n}\right)$, after $n$ observations and will assume a normal distribution given by:

$$
y_{n} \sim N\left[\theta, \frac{\sigma^{2}}{n}\right] \text {, }
$$

where $\theta$ is the underlying treatment effect that governs the trial observation. The study-specific trial result $y_{n}$ 
can estimate the true underlying treatment effect with standard error $\sigma / \sqrt{n}$ [7]. Similar to the prior discussion for the prior distribution, we can derive the equations for the likelihood (Supplemental Appendix).

\section{Conjugate Normal Model}

Given that the normal prior $\theta \sim N\left[\mu_{m}, \sigma_{m}^{2} / m_{0}\right]$ (Eq. 2) and the normal likelihood $y_{n} \sim N\left[\mu_{n}, \sigma_{n}^{2} / n\right]$ (Eq. 7) belong to the same family of mathematical functions, we have thus defined a "conjugate normal model" [7]. Derivation of the appropriate equations yields the posterior mean $\left(m_{0} \mu+n y_{n}\right) /\left(m_{0}+n\right)$ as an average of the prior mean $\mu$ and parameter estimate $y_{m}$, weighted by their respective number of observations $n$ and $m_{0}$, and is thus a compromise between the 2 [7].

\section{Bayesian Hierarchical Model}

Since the initial goal was to compare CABG with $\mathrm{PCl}$ described by 9 studies of diabetics with multivessel CAD [2,3,8-14], we believed that a hierarchical model would also be reasonable because the estimates of $\theta$ from each study might be related to each other [19]. This is modeled by viewing $\theta_{i}$ from each study as a sample from a common population distribution, which has been described in detail elsewhere [20].

\section{Software}

Under specified prior distributions, the posterior distributions of model parameters were obtained by the Gibbs sampling algorithm [21] using [R] 3.0.2 [22], BRugs [23], OpenBUGS 3.2.2 (www.openbugs.net/w/ Downloads), and Markov chain Monte Carlo methods [16]. Sample software codes are presented (Supplemental Appendix).

\section{RESULTS}

The primary outcome measure of the current study was the comparison of mortality rates at 5 years or longest follow-up after $\mathrm{CABG}$ or $\mathrm{PCl}$ in diabetic patients with multivessel CAD.

\section{Pooled Conjugate Normal Model}

Using a prior distribution elicited from the 8 trials [2,8-14] carried out before the publication of FREEDOM (Table 1) and using $\sigma=2$ (Eq. 5), we calculate the "pooled" results for the distribution by summing the $m s$ from the individual trials (Table 1) to obtain a total $m_{0}=227$. The pooled results give rise to a prior distribution with treatment effect $\mu=-0.604$ (Eq. 3), $m_{0}=227$, a standard error $\sigma / \sqrt{227}=0.133$, and

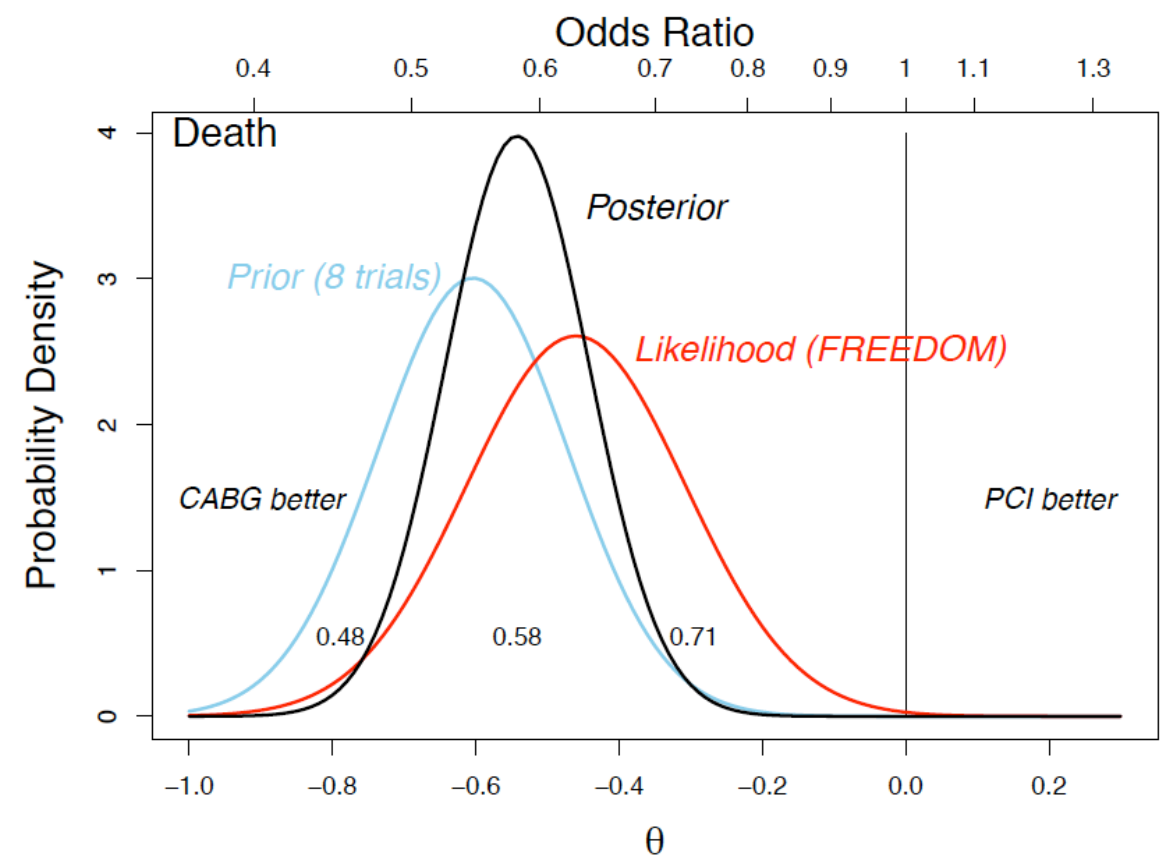

Figure 2: Bayesian Triplot Comparing Death Rates in Diabetic Patients after Coronary Artery Bypass Graft (CABG) Surgery or Percutaneous Coronary Intervention (PCl). A bell-shaped curve represents the prior distribution (blue) of possible odd ratios (ORs) based on evidence elicited from previous trials [2,8-14], and a separate curve represents the distribution of likelihood (red) obtained from the Future Revascularization Evaluation in Patients with Diabetes Mellitus: Optimal Management of Multivessel Disease (FREEDOM) [3]. Bayesian methods update the prior with the likelihood in a type of meta-analysis to produce the posterior distribution (black). The posterior is not twice the height of the prior and likelihood, because precision represented by density width is additive. The additional vertical axis intersects the origin (0.00). All probability density functions are normalized to 1 . The plot contains an $x$-axis showing the familiar $O R$, as well as an axis showing $\log _{e}(O R)$, which equals $\theta$. 
variance $V$ of $0.018=0.133^{2}$ (Eq. 4). The $95 \% \mathrm{Cl}$ for $\mu$ of -0.604 are given by $\pm 1.96 \times \sigma / \sqrt{227}= \pm 0.260$, which gives rise to values extending from -0.863 to -0.343 . Exponentiation of $\mu$ of -0.604 and its $95 \% \mathrm{Cl}$ generates values for the prior $\mathrm{OR}$ of 0.55 with $95 \% \mathrm{Cl}$ extending from 0.42 to 0.71 (Figure 2).

The likelihood calculated from FREEDOM (Table 1) gives rise to values for the OR of 0.63 with $95 \% \mathrm{Cl}$ extending from 0.47 to 0.85 (Figure 2). Because the strength of information in the likelihood is based $n=\sigma^{2} / 0.153^{2}=171$ observations, and the strength of information in the prior distribution is based on $m_{0}=\sigma^{2} / 0.133^{2}=227$ observations, the prior has approximately $m_{0} / n=227 / 171$ or $32 \%$ more information than the likelihood.

The posterior distribution (Figure 2), which is based on the equivalent number of observations in the posterior of $m_{0}+n=398$, yields an $O R$ of 0.58 with $95 \% \mathrm{BCl}$ extending from 0.48 to 0.71 . This suggests that in a pooled model, the mortality rate of diabetic patients with multivessel CAD after treatment with CABG has a $95 \%$ probability being $29 \%$ to $52 \%$ lower than that after $\mathrm{PCl}$.

\section{Conjugate Normal Model Weighted by Stent Type}

Because early studies did not incorporate newer advances in revascularization that have appeared during the past 10 years, it is reasonable to downweight older trials by adjusting the prior "number of events" from $m$ to $\alpha m$, where $\alpha$ is a normalizing factor [18]. With this approach, $\alpha$ can be subjectively set to 0 , $0.1,0.5$ and 1.0. Assigning $\alpha=0$ is equivalent to assessing the results of a previous trial as irrelevant and similar to selecting a uniform prior on the $\log _{e}(O R)$, while assigning $\alpha=1$ is equivalent to assessing full weight to the trial in the pooled analysis [18].

In a model that discounts studies by use of DES, $\alpha$ can range from 0.0 for BARI [2] to 1.0 for SYNTAX (Table 2) [13]. The model (Eq. 6) gives rise to a prior distribution with $\mu_{\text {weighted }}=-0.54$ with $95 \% \mathrm{Cl}$ extending from -0.92 to -0.15 . The $\mathrm{Cl}$ are broader in the weighted model than in the pooled model, because the effective number of observations has decreased from $m_{0}=227$ to $\alpha m_{0}=105$. Exponentiation of $\mu_{\text {weighted }}$ from the posterior distribution and its $95 \% \mathrm{BCl}$ generates an $O R_{\text {weighted }}$ of 0.61 with $95 \% \mathrm{BCl}$ extending from 0.48 to 0.78 (Figure 3).

\section{Conjugate Normal Model Weighted by Study Age}

In a model that discounts studies by year of publication, $\alpha$ can range from 0.0 for the BARI trial to 1.0 for contemporary studies (Table 2). This gives rise to a prior distribution with $\mu_{\text {weighted }}=-0.42$ with $95 \% \mathrm{Cl}$ extending from -0.74 to -0.10 , with an effective number of observations of 148 . Carrying the prior through the posterior yields an $O R_{\text {weighted }}$ of 0.64 with $95 \% \mathrm{BCl}$ extending from 0.52 to 0.80 (Figure 3 ).

Table 2: Data for Weighted Bayesian Models

\begin{tabular}{|c|c|c|c|c|c|c|}
\hline \multirow{2}{*}{ Reference } & \multirow[t]{2}{*}{ Publication year } & \multirow{2}{*}{$\begin{array}{c}\text { CABG } \\
\text { IMA Graft (\%) }\end{array}$} & \multirow{2}{*}{$\frac{\mathrm{PCl}}{\text { PTCA/BMS/DES (\%) }}$} & \multicolumn{3}{|c|}{ Study weight $(\alpha)$} \\
\hline & & & & DES & Age & LIMA \\
\hline BARI [2] & 1997 & 81 & $98 / 0 / 0$ & 0.0 & 0.0 & 0.1 \\
\hline ARTS [8] & 2001 & 93 & $11 / 89 / 0$ & 0.0 & 0.1 & 0.5 \\
\hline ERACI II [9] & 2005 & 89 & $0 / 100 / 0$ & 0.0 & 0.5 & 0.1 \\
\hline MASS II [10] & 2007 & 95 & $19 / 81 / 0$ & 0.0 & 0.5 & 1.0 \\
\hline SoS [11] & 2002 & 97 & $0 / 100 / 0$ & 0.0 & 0.1 & 1.0 \\
\hline CARDia [12] & 2010 & 94 & $0 / 31 / 69$ & 0.5 & 1.0 & 0.5 \\
\hline SYNTAX [13] & 2013 & 97 & $0 / 0 / 100$ & 1.0 & 1.0 & 1.0 \\
\hline VA CARDS [14] & 2013 & a & $0 / 0 / 100$ & 1.0 & 1.0 & 0.0 \\
\hline FREEDOM [3] & 2012 & 94 & $0 / 0 / 100$ & - & - & - \\
\hline \multicolumn{7}{|l|}{ Posterior Distribution: } \\
\hline Odds Ratio & & & & 0.61 & 0.64 & 0.64 \\
\hline $95 \% \mathrm{BCl}$ & & & & $0.48-0.78$ & $0.52-0.80$ & $0.51-0.81$ \\
\hline$m$ & & & & 105 & 148 & 125 \\
\hline
\end{tabular}

The $m$ 's are the "effective number of events" in the pooled analysis. "Arterial conduit used when possible. 

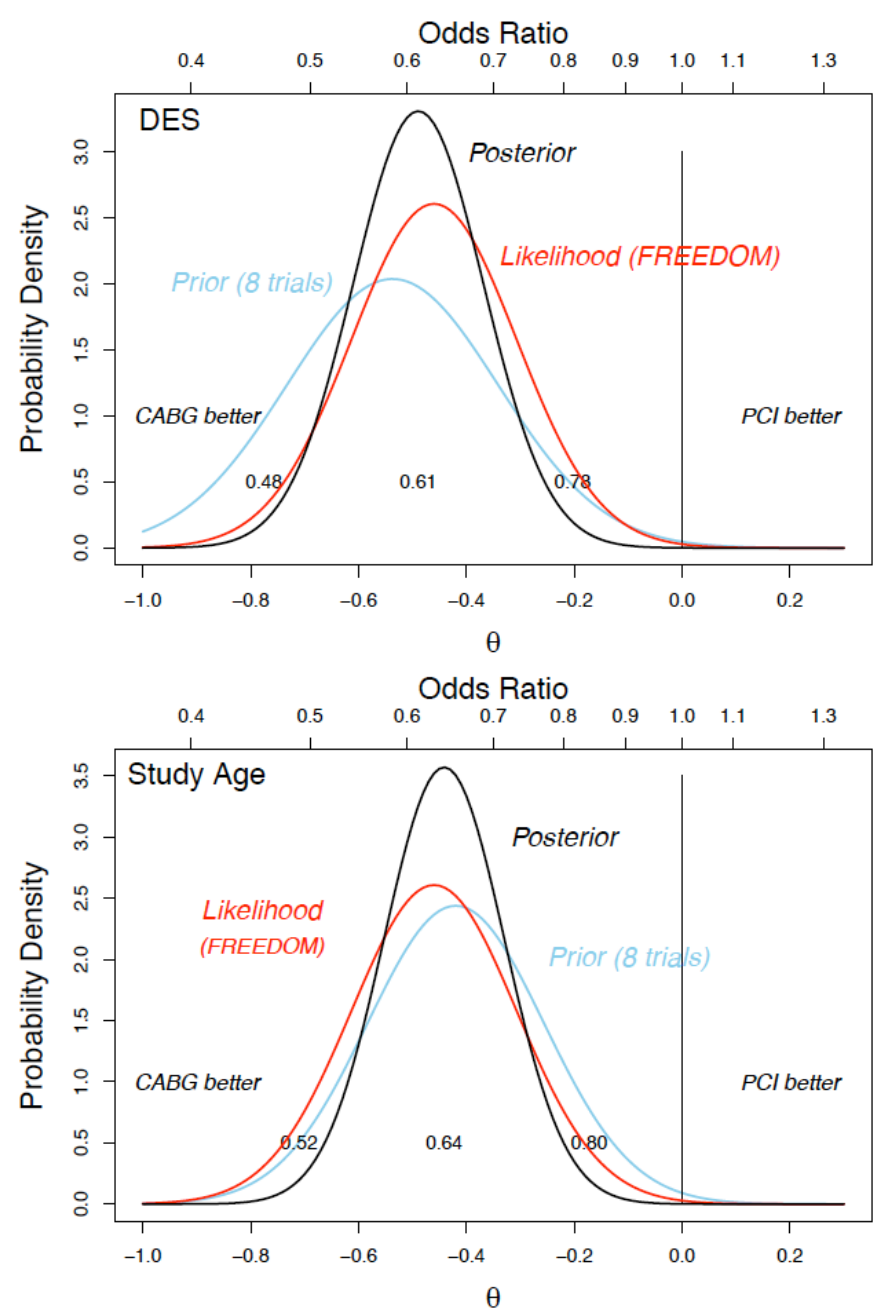

Odds Ratio

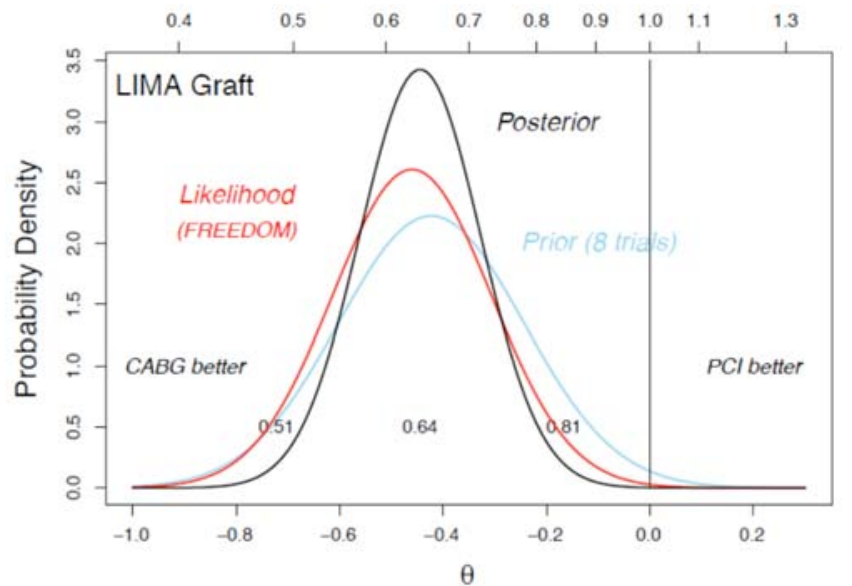

Odds Ratio

Figure 3: Bayesian Triplots Comparing Death Rates in Diabetic Patients after Coronary Artery Bypass Graft (CABG) Surgery or Percutaneous Coronary Intervention $(\mathrm{PCl})$ in a Weighted Analysis. Prior distributions (solid light blue) are elicited from 8 studies [2,8-14] and weighted by the proportion of patients receiving a drug-eluting stents (DES) during percutaneous coronary intervention, age of study, and proportion of surgical patients treated with left internal mammary (LIMA) graft. The likelihood (solid red) is obtained from the Future Revascularization Evaluation in Patients with Diabetes Mellitus: Optimal Management of Multivessel Disease trial[3]. The posterior distributions (black) contain the posterior mean odd ratios and their 95\% Bayesian credible intervals (data labels). The most obvious difference between traditional frequentist and Bayesian methods is that frequentist statistics uses only unweighted data, whereas Bayesian statistics uses both the likelihood and weighted prior information. Details of weighting are presented in Table 2. All probability density functions are normalized to 1 . The parameter $\theta$ is equivalent $\log _{\mathrm{e}}(O R)$. 


\section{Conjugate Normal Model Weighted by Use of LIMA Grafts}

In a model that discounts by the proportion of LIMA grafts used in surgical patients, $\alpha$ can range from 0.1 for studies with less than $90 \%$ use, 0.5 for $90-95 \%$ use, and 1.0 for greater than $95 \%$ use (Table 2). This gives rise to a prior distribution with $\mu_{\text {weighted }}=-0.42$ with $95 \% \mathrm{Cl}$ extending from -0.77 to -0.18 , with an effective number of observations of 125 . Carrying the prior through the posterior yields an $O R_{\text {weighted }}$ of 0.64 with $95 \% \mathrm{Cl}$ extending from 0.51 to 0.81 (Figure 3 ).

\section{Bayesian Hierarchical Meta-Analysis}

A Bayesian hierarchical meta-analysis using a noninformative prior distribution for all 9 studies produces a posterior mean odds ratio of 0.55 with $95 \% \mathrm{BCl}$ of $0.37-0.76$ (Figure 4). The broader $95 \% \mathrm{BCl}$ seen in the hierarchical model than in the normal conjugate model (0.48-0.71) represents the effect of "borrowing" from the informative prior in the normal conjugate model.

It may be instructive to compare the results of the Bayesian hierarchical meta-analysis with a traditional meta-analysis (Figure 4) of the 9 studies of patients with $\mathrm{DM}$ and multivessel CAD ( $\mathrm{N}=3,484$ patients). The latter approach using a random effects model produces a pooled odds ratio and $95 \%$ confidence interval favoring the use of $\mathrm{CABG}$ over $\mathrm{PCl}$ to improve survival $(0.54,0.38-0.76)$, in good agreement with the
Bayesian hierarchical meta-analysis using a noninformative prior distribution $(0.54,0.38-0.76)$

\section{DISCUSSION}

The issue of identifying whether CABG is superior to $\mathrm{PCl}$ for diabetic patients with multivessel $\mathrm{CAD}$ is larger than the results from an individual trial or a metaanalysis of several trials. A Bayesian approach uses the process of inductive inference, which is similar to the way that clinicians make a diagnosis from clinical evidence at the bedside [24]. Clinicians know, for example, that an elevated troponin does not always equate with myocardial infarction [25]. The Bayesian approach treats clinical trial results like a troponin elevation and puts new trial results (ie, $O R$ ) into the context of what is already known from previous studies to define the probability distribution for the parameter $\theta$ that governs the underlying treatment effect (eg, lower mortality after CABG than after PCl).

\section{Clinical Guidelines}

The current American College of Cardiology Foundation/American Heart Association (ACCF/AHA) guideline states that [26]:

Class I - CABG is generally recommended in preference to $\mathrm{PCl}$ to improve survival in patients with diabetes mellitus and multivessel CAD for which revascularization is likely to improve survival (3-vessel

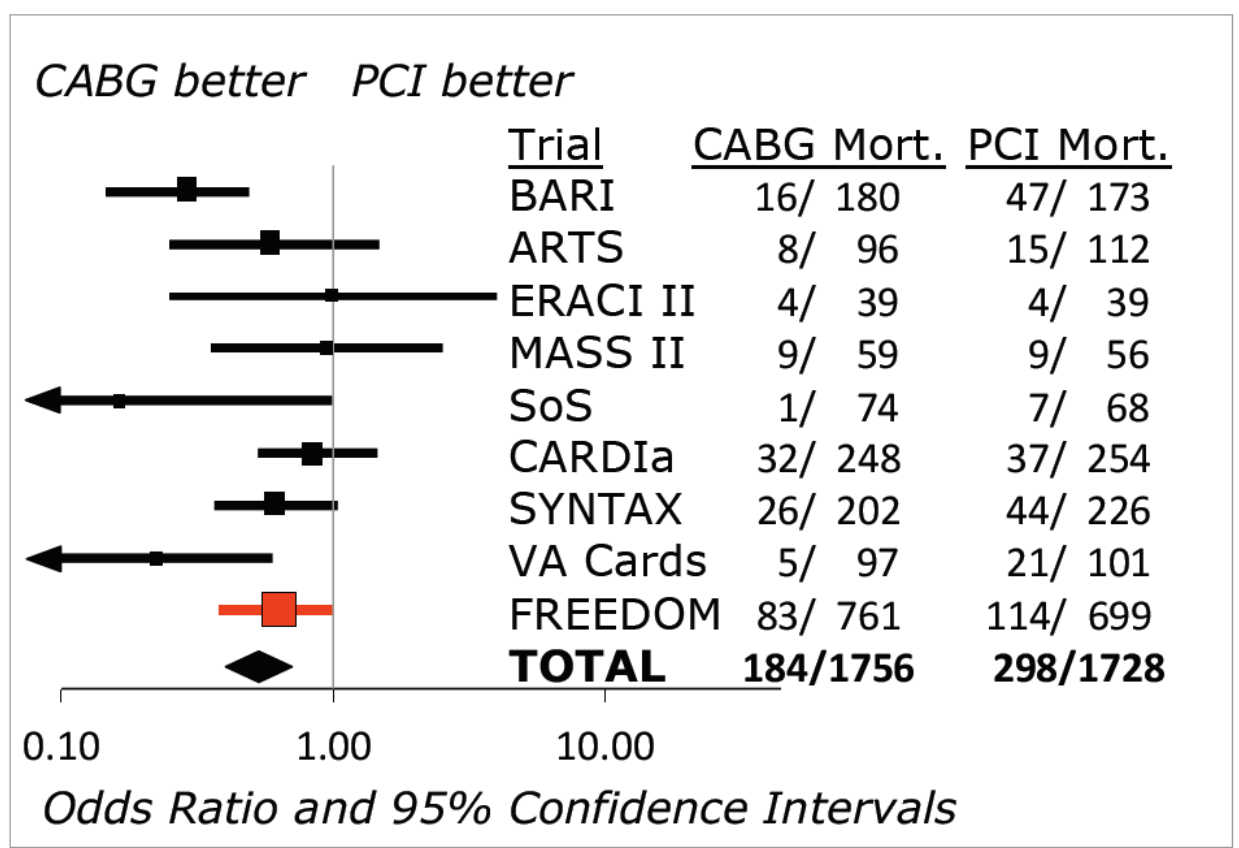

Figure 4: Forest Plot of Studies Comparing Coronary Artery Bypass Graft (CABG) Surgery with Percutaneous Coronary Intervention (PCI) in Diabetic Patients with Multivessel Coronary Artery Disease. 
CAD or complex 2-vessel CAD involving the proximal LAD), particularly if a LIMA graft can be anastomosed to the LAD artery, provided the patient is a good candidate for surgery. (Level of Evidence: B)

A Class I recommendation for CABG in diabetics with multivessel coronary artery disease suggests that the benefits clearly outweigh risk in most, if not all, diabetic patients with multivessel CAD. A suggestion of a positive result in overall survival in FREEDOM [3] signifies that $C A B G$ appeared to confer greater survival than did $\mathrm{PCl}$ in a single cohort of patients at a single point in time.

\section{FREEDOM}

The FREEDOM trial was a dedicated trial in a diabetic population with multivessel CAD designed test the hypothesis that CABG is superior to $\mathrm{PCl}$. The primary endpoint of all-cause death $\mathrm{MI}$, or stroke occurred less frequently in the CABG group than in the $\mathrm{PCl}$ group $(18.7 \%$ vs. $26.6 \%, P=0.005)$. The benefit of CABG was driven by differences in rates of both myocardial infarction $(P<0.001)$ and death from any cause $(P=0.049)$. The significance of the survival advantage for CABG has remained controversial, however, because a final $P$ value of less than 0.044 was considered to indicate statistical significance for the primary outcome after 3 interim analyses had been performed [3].

\section{Current Analysis}

The current analysis used several Bayesian approaches. The results of the normal conjugate model favored the use of CABG over PCI for diabetic patients with multivessel CAD. In a sensitivity analysis, models weighted by the use of DES, study age and use of arterial conduits also favored the use of CABG over $\mathrm{PCl}$ for diabetic patients with multivessel CAD.

\section{LIMITATIONS}

Limitations of the current study include the possibility of "hindsight bias," because we assessed the prior distribution after seeing the report of Verma and colleagues [4] and the 2011 clinical guidelines for revascularization [15]. However, we carried out the current analysis prospectively and concurrently with the creation of the 2014 focused update of the guidelines for the diagnosis and management of patients with stable ischemic heart disease [26]. To complete the analysis, we assumed that the RCTs were exchangeable [29], because the studies were of the same fundamental design and all trial and subgroups analyses dealt with diabetic patients with multivessel CAD. Another potential limitation of the current analysis involves the changes in treatments introduced during the past 20 years in which the various RCTs were conducted. For example, BARI used PTCA [2], 4 studies used bare-metal stents (BMS) [8-11], and 4 studies used DES [3, 7, 12-14]. We dealt with the issue of multiplicity of treatments by performing sensitivity analysis. The subjective nature of the weighting parameter $\alpha$ used in the sensitivity analysis is also recognized. Accordingly, we recognize that the sensitivity analyses involving DES, study age and the use of the LIMA grafts must be considered exploratory.

\section{SUPPLEMENTAL MATERIALS}

The supplemental materials can be downloaded from the journal website along with the article.

\section{REFERENCES}

[1] Roffi M, Brandle M. Diabetes. In: Topol EJ, Teirstein PS, editors. Textbook of Interventional Cardiology. $6^{\text {th }}$ ed. Philadelphia: Saunders Elsevier 2012; pp. 30-49. http://dx.doi.org/10.1016/B978-1-4377-2358-8.00003-6

[2] The BARI Investigators. Influence of diabetes on 5-year mortality and morbidity in a randomized trial comparing CABG and PTCA in patients with multivessel disease: the Bypass Angioplasty Revascularization Investigation (BARI). Circulation 1997; 96: 1761-9.

http://dx.doi.org/10.1161/01.CIR.96.6.1761

[3] Farkhouh ME, Domanski M, Sleeper LA, et al. Strategies for multivessel revascularization in patients with diabetes. $\mathrm{N}$ Engl J Med 2012; 367: 2375-2384. http://dx.doi.org/10.1056/NEJMoa1211585

[4] Verma S, Farkouh ME, Yanagawa B, et al. Comparison of coronary artery bypass surgery and percutaneous coronary intervention in patients with diabetes: a meta-analysis of randomised controlled trials. Lancet Diabetes Endocrinol 2013; 1: 317-328.

http://dx.doi.org/10.1016/S2213-8587(13)70089-5

[5] Bangalore S, Toklu B, Feit F. Outcomes with coronary artery bypass graft surgery versus percutaneous coronary intervention for patients with diabetes mellitus: Can newer generation drug-eluting stents bridge the gap? Circ Cardiovasc Interv 2014

[6] Hakeem A, Garg N, Bhatti S, Rajpurohit N, Ahmed Z, Uretsky BF. Effectiveness of percutaneous coronary intervention with drug-eluting stents compared with bypass surgery in diabetics with multivessel coronary disease: comprehensive systematic review and meta-analysis of randomized clinical data. J Am Heart Assoc 2013; 2: e000354. http://dx.doi.org/10.1161/JAHA.113.000354

[7] Spiegelhalter DJ, Abrams KR, Myles JP. Bayesian Approaches to Clinical Trials and Health Care Evaluations. Chichester, England: Wiley 2004.

[8] Abizaid A, Costa MA, Centemero M, et al. Clinical and economic impact of diabetes mellitus on percutaneous and surgical treatment of multivessel coronary disease patients: insights from the Arterial Revascularization Therapy Study (ARTS) trial. Circulation 2001; 104: 533-538. http://dx.doi.org/10.1161/hc3101.093700 
[9] Rodriguez AE, Baldi J, Fernandez Pereira C, et al. Five-year follow-up of the Argentine randomized trial of coronary angioplasty with stenting versus coronary bypass surgery in patients with multiple vessel disease (ERACI II). J Am Coll Cardiol 2005; 46: 582-8. http://dx.doi.org/10.1016/j.jacc.2004.12.081

[10] Hueb W, Gersh BJ, Costa F, et al. Impact of diabetes on fiveyear outcomes of patients with multivessel coronary artery disease. Ann Thorac Surg 2007; 83: 93-9. http://dx.doi.org/10.1016/j.athoracsur.2006.08.050

[11] The SoS Investigators. Coronary artery bypass surgery versus percutaneous coronary intervention with stent implantation in patients with multivessel coronary artery disease (the Stent or Surgery trial): a randomised controlled trial. Lancet 2002; 360: 965-70. http://dx.doi.org/10.1016/S0140-6736(02)11078-6

[12] Kapur A, Hall RJ, Malik IS, et al. Randomized comparison of percutaneous coronary intervention with coronary artery bypass grafting in diabetic patients. 1-year results of the CARDia (Coronary Artery Revascularization in Diabetes) trial. J Am Coll Cardiol 2010; 55: 432-440. http://dx.doi.org/10.1016/j.jacc.2009.10.014

[13] Kappetein AP, Head SJ, Morice M-C, et al. Treatment of complex coronary artery disease in patients with diabetes: 5year results comparing outcomes of bypass surgery and percutaneous coronary intervention in the SYNTAX trial. Eur J Cardiothorac Surg 2013; 43: 1006-1013. http://dx.doi.org/10.1093/ejcts/ezt017

[14] Kamelesh $M$, Sharp TG, Tang XC, et al. Percutaneous coronary intervention versus coronary bypass surgery in United States veterans with diabetes. J Am Coll Cardiol 2013; 61: 808-816. http://dx.doi.org/10.1016/j.jacc.2012.11.044

[15] Levine GN, Bates ER, Blankenship JC, et al. 2011 ACCF/AHA/SCAI guidelines for percutaneous coronary intervention: A report of the American College of Cardiology Foundation/American Heart Association Task Force on Practice Guidelines and the Society for Cardiovascular Angiography and Interventions. Circulation 2011: e574-e65 plus Data Supplement 1.

[16] Lunn D, Jackson C, Best N, Thomas A, Spiegelhalter D. The BUGS Book: A Practical Introduction to Bayesian Analysis. Boca Raton, FL: CRC Press 2013.

[17] Albert J. Bayesian Computation with R. New York, NY: Springer Science+Business Media, LLC 2007.

[18] Ibrahim JG, Chen M-H. Power prior distributions for regression models. Stat Sci 2000; 15: 46-60. http://dx.doi.org/10.1214/ss/1009212673

[19] Gelman A, Carlin JB, Stern HS, Rubin DB. Bayesian Data Analysis. Boca Raton, FL: Chapman and Hall/CRC 2013.

[20] He Y, Bittl JA, Wouhib A, Normand S-LT. Case study in cardiovascular medicine: unprotected left main coronary artery disease. In: Biondi-Zoccai G, editor Network MetaAnalysis: Evidence Synthesis with Mixed Treatment Comparison. New York: Nova Science Publishers, Inc. 2014.

[21] Gelfand AE, Smith AFM. Sampling-based approaches to calculate marginal densities. J Am Stat Assoc 1990; 85: 398409.

http://dx.doi.org/10.1080/01621459.1990.10476213
[22] R Core Team. R: A language and environment for statistical computing. In: R Foundation for Statistical Computing. Vienna, Austria 2013.

[23] Thomas A, O'Hara B, Ligges U, Sturtz S. Making BUGS Open. R News 2006.

[24] Goodman SN. Toward evidence-based medical statistics. 1: the P value fallacy. Ann Int Med 1999; 130: 995-1004. http://dx.doi.org/10.7326/0003-4819-130-12-199906150$\underline{00008}$

[25] Diamond GA, Kaul S. How would the Reverend Bayes interpret high-sensitivity troponin? Circulation 2010; 121: $1172-1175$.

http://dx.doi.org/10.1161/CIR.0b013e3181d839e8

[26] Fihn SD, Blankenship JC, Alexander KP, et al. 2014 ACCF/AHA/AATS/PCNA/SCAI/STS focused update of the guidelines for the diagnosis and management of patients with stable ischemic heart disease: a report of the Amercan College of Cardiology/American Heart Association Task Force on Practice Guidelines, and the American Association for Thoracic Surgery, Preventive Cardiovascular Nurses Association, Society for Cardiovascular Angiography and Interventions, and Society of Thoracic Surgeons. Circulation 2014; 130: 1749-1767.

\section{http://dx.doi.org/10.1161/CIR.0000000000000095}

[27] Armitage P, Berry G, Matthews JNS. Clinical trials. Statistical Methods in Clinical Research. Malden, MA: Blackwell Science 2002; pp. 591-647.

[28] DerSimonian R, Laird N. Meta-analysis in clinical trials. Control Clin Trials 1986; 7: 177-188. http://dx.doi.org/10.1016/0197-2456(86)90046-2

[29] Lumley T. Network meta-analysis for indirect treatment comparisons. Stat in Med 2002; 21: 2313-2324. http://dx.doi.org/10.1002/sim.1201

[30] Brophy JM, Belisle P, Joseph L. Evidence for use of coronary stents. A hierarchical bayesian meta-analysis. Ann Int Med 2003; 138: 777-786

http://dx.doi.org/10.7326/0003-4819-138-10-200305200$\underline{00005}$

[31] Al Suwaidi J, Holmes DR Jr, Salam AM, Lennon R, Berger PB. Impact of coronary artery stents on mortality and nonfatal myocardial infarction: meta-analysis of randomized trials comparing a strategy of rountine stenting with that of balloon angioplasty. Am Heart J 2004; 147: 756-758. http://dx.doi.org/10.1016/j.ahj.2003.11.025

[32] Kastrati A, Mehilli J, Pache J, et al. Analysis of 14 trials comparing sirolimus-eluting stents with bare-metal stents. $\mathrm{N}$ Engl J Med 2007; 356: 1030-1039. http://dx.doi.org/10.1056/NEJMoa067484

[33] Trikalinos TA, Alsheikh-Ali AA, Tatsioni A, Nallamothu BK, Kent DM. Percutaneous coronary interventions for non-acute coronary artery disease: a quantitative 20-year synopsis and a network meta-analysis. Lancet 2009; 373: 911-918. http://dx.doi.org/10.1016/S0140-6736(09)60319-6

Received on 24-11-2014

Accepted on 30-12-2014

Published on 27-01-2015

http://dx.doi.org/10.6000/1929-6029.2015.04.01.3

(C) 2015 Lang et al.; Licensee Lifescience Global.

This is an open access article licensed under the terms of the Creative Commons Attribution Non-Commercial License (http://creativecommons.org/licenses/by-nc/3.0/) which permits unrestricted, non-commercial use, distribution and reproduction in any medium, provided the work is properly cited. 